\title{
Auto-Cross-Linking Hydrogels of Hydrogen Peroxide-Oxidized Pectin and Gelatin for Applications in Controlled Drug Delivery
}

\author{
Claudia A. Garrido, ${ }^{1}$ Michel Vargas, ${ }^{2}$ and Jose F. Alvarez-Barreto $\mathbb{D}^{1}$ \\ ${ }^{1}$ Biomaterials Laboratory, Instituto para el Desarrollo de Energias y Materiales Alternativos, Department of Chemical Engineering, \\ Universidad San Francisco de Quito, Quito 170157, Ecuador \\ ${ }^{2}$ Department of Mechanical Engineering, Universidad San Francisco de Quito, Quito 170157, Ecuador
}

Correspondence should be addressed to Jose F. Alvarez-Barreto; jalvarezb@usfq.edu.ec

Received 19 September 2018; Accepted 23 December 2018; Published 24 February 2019

Academic Editor: Matthias Schnabelrauch

Copyright (c) 2019 Claudia A. Garrido et al. This is an open access article distributed under the Creative Commons Attribution License, which permits unrestricted use, distribution, and reproduction in any medium, provided the original work is properly cited.

\begin{abstract}
Pectin-based hydrogels for biomedical applications have attracted recent attention due to their low cost, large availability of the materials, and high levels of biocompatibility. Specifically, periodate-oxidized pectin has been combined with chitosan and gelatin to form different structures. However, hydrogen peroxide-mediated oxidation of pectin has not been studied for this application; furthermore, there is little information on the effect of the degree of oxidation on hydrogel characteristics nor has the feasibility of these systems as controlled drug delivery matrices been explored. Thus, the present work proposes to study the properties of gelatin-peroxide-oxidized pectin hydrogels as drug delivery systems in wound dressing applications. Combinations of pectin at different degrees of oxidation (high, low, and native pectin) and gelatin were analyzed and tested by swelling properties, reswelling from xerogel and aerogel forms, SEM, FTIR, and drug release. It was determined that hydrogels that contained oxidized pectin had improved swelling ratios and stability, at $32^{\circ} \mathrm{C}$, compared to those with native pectin and only gelatin. The porosity of the oxidized pectin hydrogels allowed to have sustained and high release rates, which would make them an attractive alternative for wound dressings.
\end{abstract}

\section{Introduction}

Natural polysaccharides, like pectin and gelatin, are used in the formation of hydrogels for different biomedical applications, such as drug delivery and tissue engineering due to their high biocompatibility, low toxicity, and biodegradability [1]. Controlled drug delivery refers to the technologies and systems that allow a drug to better perform its effect [2]; encapsulation in hydrogels is an alternative that reduces side effects, directs the drug towards a target, and moderates the reaction time [3].

Pectin, a polysaccharide also called polygalacturonic acid, is a major component of plant cell walls [4] and can be obtained through an acid extraction procedure, or microwave-assisted extraction, among other methods. The importance of this polysaccharide relies on its various applications in the food industry as a thickening agent and texture modifier, as well as applications in the cosmetic and textile industries [5].

Gelatin, on the other hand, is a naturally occurring protein derived from the hydrolysis of collagen; its composition is complex, mainly consisting of several proteins that come from collagen which grants properties beyond its culinary use, incurring in pharmaceutical or medical uses or in the generation of materials with potential for the engineering of ocular tissues [6]. Gelatin is a colloid rich in amino and carboxylic groups, which allow chemical cross-linking with other polysaccharides. These properties make it possible to create several products such as cellular adhesives for tissue regeneration, resulting from gelatin cross-linking with glutaraldehyde to form hydrogels [7], and protein release matrices [8], among other applications.

Gelatin and pectin are biomaterials that can be used in drug transport. Currently, pectin has been used to treat colon 
cancer [9] and form different systems such as nanoparticles, films, and matrices for several uses [10]. In addition to that, studies on gelatin have focused on its use as a possible biomaterial in tissue engineering [11].

As previously mentioned, pectin and gelatin are biomaterials which can be used independently, but there are also cross-linking systems which involve their functionalization in order to create a new material having novel characteristics. Investigations of gelatin as a hydrogel have been performed employing cross-linking agents such as glutaraldehyde, which substantially improves its mechanical properties such as Young's modulus, thermal stability, and swelling; however, it is a highly toxic agent at high concentrations [12]. On the other hand, pectin has been cross-linked with ethylene glycol diglyceryl and glutaraldehyde, creating gels with high absorption capacity and good characteristics for loading of drugs [13]. However, these cross-linking agents could potentially hinder the application of these systems due to toxicity concerns. One of the most recent investigations is with oxidized pectin, gelatin, and glycerol as a cross-linking agent, which would not require the use of glutaraldehyde [7].

The objective of the present investigation is to study the properties of gelatin-oxidized pectin hydrogels, without glycerol, as drug delivery systems. It is important to point out that gelatin and oxidized pectin (OP) with OP as a cross-linker have been previously studied, using sodium periodate as the oxidizing agent, but were not evaluated as drug carriers [14]. However, the present work proposes an alternative pectin oxidation method, using hydrogen peroxide as the oxidizing agent, which is cheaper, less toxic, and more efficient for polymer modification [15]. Due to all the properties hydrogen peroxide has, the oxidation procedure is less time-consuming, and lower temperatures and concentrations of the oxidizing agent are required. Furthermore, additional tests were performed for gelatin-OP hydrogels, like swelling, dry swelling, and drug loading properties, features that had not been explored previously and that allow assessment of the feasibility of the system for possible applications.

\section{Materials and Methods}

2.1. Pectin Oxidation. The following methodology is based on the modification procedure reported by Gupta et al. on pectin oxidation with periodic acid [7]. In this work, pectin oxidation was carried out using a different oxidizing agent, hydrogen peroxide. Two kinds of oxidized pectin were used, which differed in their degree of oxidation, and the conditions for the modification were established through extensive preliminary assays.

For the preparation of high-oxidation pectin (HOP) (aldehyde content greater than 0.25 moles of aldehyde per gram), $50 \mathrm{~mL}$ of a $10 \% w / v$ aqueous pectin solution was moderately stirred and $50 \mathrm{~mL}$ of $30 \% v / v$ hydrogen peroxide $\left(\mathrm{H}_{2} \mathrm{O}_{2}\right)$ was added. The oxidation was carried for $30 \mathrm{~min}$, at $\mathrm{pH}$ 1, room temperature, and protected from the light.

For the preparation of low-oxidation pectin (LOP) (aldehyde content below 0.25 moles of aldehyde per gram), $71.7 \mathrm{~mL}$ of a $10 \% \mathrm{w} / \mathrm{v}$ aqueous pectin solution was moderately stirred and $28.3 \mathrm{~mL}$ of $30 \% v / v \mathrm{H}_{2} \mathrm{O}_{2}$ solution was
TABLE 1: Summary of the conditions for pectin oxidation.

\begin{tabular}{lcc}
\hline Conditions of oxidation & $\begin{array}{c}\text { Low-oxidation pectin } \\
\text { (LOP) }\end{array}$ & $\begin{array}{c}\text { High-oxidation } \\
\text { pectin (HOP) }\end{array}$ \\
\hline Temperature $\left({ }^{\circ} \mathrm{C}\right)$ & 25 & 25 \\
Reaction time $(\mathrm{min})$ & 165 & 30 \\
$\mathrm{pH}$ & 2 & 1 \\
$\mathrm{H}_{2} \mathrm{O}_{2}$ concentration $(\%)$ & 8.5 & 15 \\
\hline
\end{tabular}

TABle 2: Summary of hydrogel compositions. The percentages of pectin and gelatin weight were according to the total volume of the solution (e.g., in $10 \mathrm{~mL}$ of distilled water, $2.0 \mathrm{~g}$ of gelatin and $0.6 \mathrm{~g}$ of pectin are dissolved).

\begin{tabular}{lcccc}
\hline $\begin{array}{l}\text { Components of the } \\
\text { hydrogel }\end{array}$ & H.6.20 & L.6.20 & N.6.20 & G.0.20 \\
\hline $\begin{array}{l}\text { Type of pectin } \\
\text { Gelatin }(w / v \%)\end{array}$ & HOP & LOP & Native (NP) & No pectin \\
Pectin $(w / v \%)$ & 20 & 20 & 20 & 20 \\
\hline
\end{tabular}

added. The oxidation was carried for $165 \mathrm{~min}$, at $\mathrm{pH} 2$, room temperature, and protected from the light.

The oxidized pectin was precipitated, using 96\% ethanol, filtered, and dried at $40^{\circ} \mathrm{C}$ until constant weight. Table 1 summarizes the oxidation conditions for both types of pectin.

2.2. Determination of Aldehyde Groups. In order to differentiate HOP and LOP, the aldehyde content was quantified, according to the procedure proposed by Zhao and Heindel, based on a colorimetric titration [16]. First, a hydroxylamine $0.25 \mathrm{~N}$ hydrochloride solution was prepared by dissolving the reagent in $1000 \mathrm{~mL}$ of distilled water and $6.0 \mathrm{~mL}$ of methyl orange $(0.05 \%)$; then, $\mathrm{pH}$ was adjusted to 4 . After that, $0.1 \mathrm{~g}$ of the sample was dissolved in $25 \mathrm{~mL}$ of the previous solution and left stirring for 2 hours. Finally, it was titrated with standardized $0.1 \mathrm{~N} \mathrm{NaOH}$. This procedure was made for aldehyde quantification in dextran [16]; however, an exact molecular weight is required, and therefore, a calibration curve using 2-methylbutanal as a standard was used to calculate the aldehyde content per gram of sample.

2.3. Hydrogel Formation. The hydrogels were formed through a modification of methodology proposed by Mishra et al., which refers to the hydrogel formation of pectin gelatin membranes with glutaraldehyde [17]. However, the hydrogels proposed in the current study do not require an additional chemical cross-linker, since the oxidized pectin plays that role.

In this case, the hydrogel formation required several combinations of concentrations of pectin and gelatin but also different degrees of oxidation of pectin (high, low, and native), as shown in Table 2; also, a blank hydrogel made of sole gelatin was prepared. In general, for any combination, a determined amount of pectin was mixed with gelatin; then, this powder mixture was added to $10 \mathrm{~mL}$ of water, and the $\mathrm{pH}$ was adjusted to 6 and left stirring. After 20 minutes, the 
solution was placed in molds of $1 \mathrm{~cm}$ of diameter and left to cross-link for 24 hours.

2.4. Hydrogel Swelling. The swelling ratio methodology was based on the one reported by Begam et al. [18]. The swelling rate was measured by the weight variations that occurred when the pectin-gelatin hydrogels were left in phosphate-buffered saline ( $\mathrm{pH} 7.4)$.

First, $1 \mathrm{~L}$ of the solution of phosphate-buffered saline (PBS) was prepared using $8.0 \mathrm{~g}$ of $\mathrm{NaCl}, 0.2 \mathrm{~g}$ of KCL, $1.42 \mathrm{~g}$ of $\mathrm{Na}_{2} \mathrm{HPO}_{4}$, and $0.24 \mathrm{~g} \mathrm{KH}_{2} \mathrm{PO}_{4}$. It was stored in glass containers at $4^{\circ} \mathrm{C}$ and warmed to room temperature before using.

Hydrogels were prepared as previously explained and placed in 24-well plates with $10 \mathrm{~mL}$ of PBS, followed by incubation at $32^{\circ} \mathrm{C}$. The samples were weighed periodically for 48 hours. For this test, all possible variations of pectin content $(2 \%, 4 \%$, and $6 \%)$, degree of oxidation of pectin (high, low, and native pectin), and gelatin content $(10 \%, 15 \%$, and $20 \%$ ) of hydrogels were assessed in order to determine the most stable ones. Equation (1) allows calculation of the swelling rate as a percentage of the initial weight, a value that is shown in the following swelling rate figures

$$
\% S=\frac{W_{t}-W_{0}}{W_{0}} \times 100
$$

2.5. Xerogel and Aerogel Swelling. The procedure for xerogel and aerogel swelling was based on the methodology proposed by Patel and Amiji [19]. The dry swelling rate was performed similar to the swelling rate previously mentioned, but for this procedure, hydrogels were dried by two methods: dried overnight in an oven at $35^{\circ} \mathrm{C}$ (xerogel) and dried by lyophilization (aerogel). Then, the samples were placed in 6-well plates with $10 \mathrm{~mL}$ of PBS, $\mathrm{pH} 7.4$, and were weighed periodically, for 80 hours. Equation (1) was used for determining the xerogel swelling.

2.6. Drug Loading. The drug loading process was based on the ones reported by Gaihre et al. on drug loading through desolvation/cross-linking on gelatin-coated magnetic iron oxide nanoparticles [20]. For loading and release analysis, an acetaminophen standard (Sigma-Aldrich, catalogue number A3035) was used. In this case, hydrogels were formed in the presence of the drug, with a loading of $25 \mathrm{w} \%$ of the gelatin weight. Acetaminophen, pectin, and gelatin were weighed together and dissolved in water as one batch in $10 \mathrm{~mL}$ of distilled water and stirred for 20 minutes. Then, approximately $1 \mathrm{~mL}$ of the previous hydrogel mixture was placed in $1 \mathrm{~cm}$ diameter molds and cross-linked for 24 hours. The amount of acetaminophen per hydrogel was $250 \mathrm{mg}$ ( $1.0 \mathrm{~g}$ of gelatin and $0.6 \mathrm{~g}$ of $\mathrm{OP}$ ), a dose that allowed gelatin cross-linking according to previous studies on gelatin systems [21].

2.7. Drug Release. Drug release methodology was based on the methodology proposed by Kumar et al. for drug release studies in pectin-chitin/nano $\mathrm{CaCO}_{3}$ composite scaffolds [22]. Release studies were performed in simulated body fluid (SBF) media at $32^{\circ} \mathrm{C}$. The hydrogels were weighed to determine the total amount of drug that could be released; then, they were submerged in $10 \mathrm{~mL}$ of SBF (constant volume), and aliquots of $125 \mu \mathrm{L}$ were extracted and then replenished with fresh SBF. The aliquots were diluted to $25 \mathrm{~mL}$ using the same solution of SBF. Acetaminophen quantification was carried by spectrophotometric analysis at $240 \mathrm{~nm}$, using the equipment CECIL 2041. A standard curve was made with solutions of known concentrations, and the percentage of the released drug $\left(\% D_{\mathrm{r}}\right)$ is determined according to

$$
\% D_{\mathrm{r}}=\frac{D_{t}-D_{t-1}}{D_{0}} \times 100,
$$

where $D_{t}$ is the amount in mg of drug released in time $t, D_{t-1}$ is the cumulative amount of drug released during the release period, and $D_{0}$ is the initial drug weight.

2.8. FTIR Spectroscopy. Infrared spectra were taken using a Cary 630 FTIR Spectrometer from Agilent Technologies.

2.9. Scanning Electron Microscopy. Hydrogel morphological analyses were carried out in a JEOL JSM-IT300 Scanning Electron Microscope (Tokyo, Japan). Samples were lyophilized and placed on metallic stubs with a carbon tape. Images were obtained at a potential of $5 \mathrm{kV}$ and $30 \mathrm{~Pa}$.

2.10. Statistical Analysis. For all assays, four samples were tested $(n=4)$, unless otherwise specified. Results were reported as average \pm standard deviation. A two-way ANOVA test was applied, and multiple pair-wise comparisons were carried out using the Tukey-HSD method with a $95 \%$ confidence level $(p \leq 0.5)$.

\section{Results and Discussion}

Hydrogels were fabricated using the previously mentioned procedure, in which the oxidized pectin would act as a cross-linker to the gelatin matrix. The following hydrogels were tested for wound dressing systems at $32^{\circ} \mathrm{C}[23]$, as previous tests were performed at $37^{\circ} \mathrm{C}$ and no thermal stability was found.

Initially, it was important to assess different compositions in order to define working conditions, later identified as those reported in Table 2, for more thorough hydrogel characterization. Different gelatin compositions (from 5 to $20 \mathrm{w} \%)$ and pectin concentrations (0 to $6 \mathrm{w} \%)$ were assayed. Figure 1 shows an example of some of these formulations varying both concentrations, using HOP. From these, it can be seen that the swelling degree increased with gelatin concentrations; at $20 \mathrm{w} \%$ gelatin, values of up to $140 \%$ in swelling were obtained, while at $10 \mathrm{w} \%$ gelatin, all values were below $100 \%$. In some cases, signs of degradation were observed, represented by decreases in swelling after a certain point. This is the case for the $4 \%$ HOP- $15 \%$ gelatin and $2 \%$ HOP-15\% gelatin formulations. Overall, the hydrogels based on $20 \%$ gelatin, with different HOP concentrations, were more stable and achieved higher degrees of swelling. Additionally, for all gelatin compositions, the hydrogels with $6 \%$ HOP were consistently stable, achieving swelling equilibrium after $72 \mathrm{~h}$. Similar behaviors were observed with LOP and NOP. Therefore, the remaining results and analysis in this 


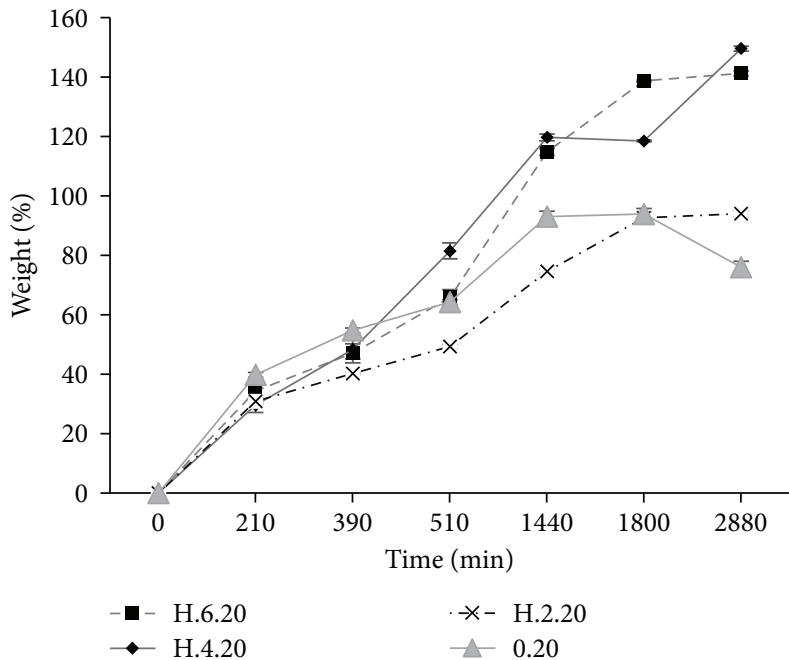

(a)

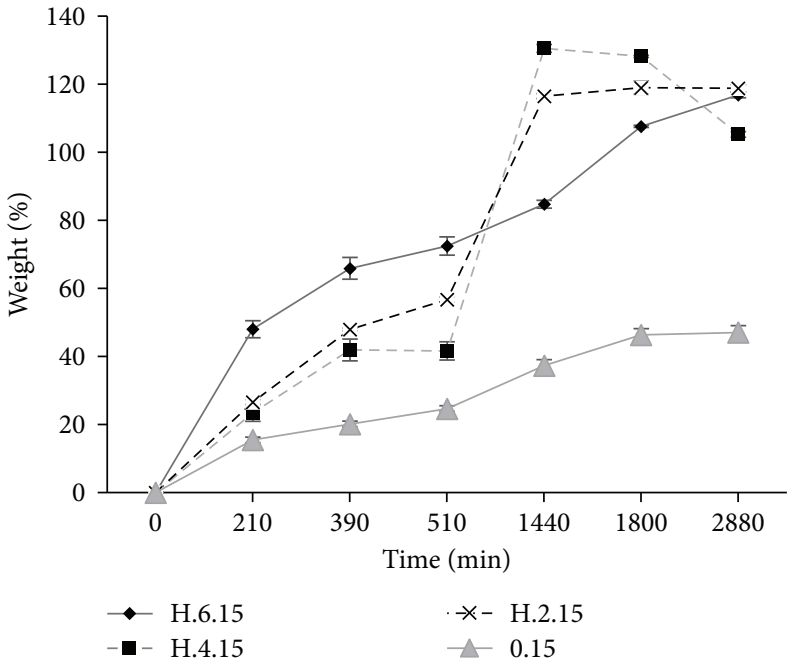

(b)

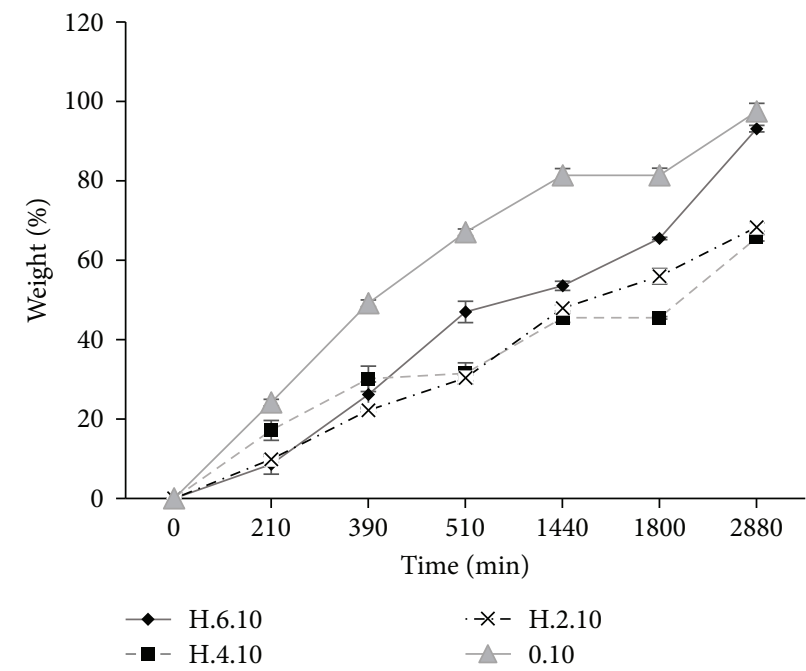

(c)

FIGURE 1: Swelling kinetics for different formulations of hydrogels incubated in PBS. All samples were prepared with HOP figures which are variations in gelatin concentration: (a) OP hydrogels with $20 \%$ gelatin, (b) OP hydrogels with 15\% gelatin, and (c) OP hydrogels with $10 \%$ gelatin. The first number in the legend indicates the HOP composition, and the second number corresponds to the gelatin concentration, i.e., $\mathrm{H} 4.10$ is the hydrogel with $4 \% \mathrm{HOP}$ and $10 \%$ gelatin.

study were carried out using $20 \%$ gelatin and $6 \%$ pectin of different oxidation levels.

Figure 2 shows the different formulations of the hydrogels freshly made. As it can be seen, the presence of pectin increases the turbidity of the hydrogel as the particles of pectin are suspended in the matrix [24]; on the other hand, gelatin appeared to form a homogeneous hydrogel.

3.1. Swelling. Figure 3 shows the swelling studies and the different behaviors among the variety of pectin. Low-oxidation pectin had the highest swelling ratio $(181.33 \pm 3.05 \%)$, and its swelling equilibrium was reached after the second day (2880 minutes). The lowest swelling ratio was displayed by gelatin alone, with swelling equilibrium, at a value of 93.90 $\pm 1.84 \%$, after $24 \mathrm{~h}$. HOP and NOP had similar behaviors in terms of swelling. Pectin, either modified or native,

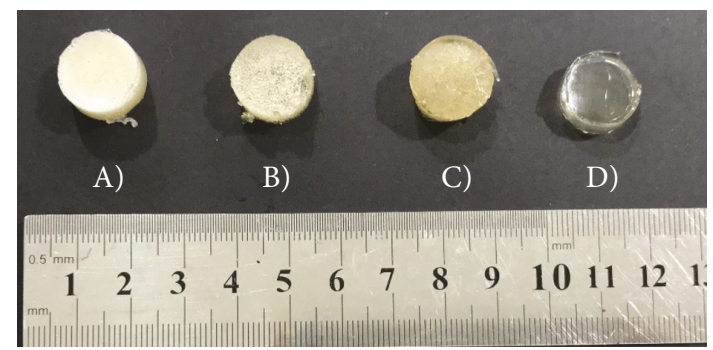

Figure 2: Hydrogels of gelatin and pectin, with different oxidation levels, obtained after $24 \mathrm{~h}$ of cross-linking. The samples shown are (A) H.6.20 (6\% HOP and 20\% of gelatin), (B) L.6.20 (6\% LOP and $20 \%$ of gelatin), (C) N.6.20 (6\% NOP and $20 \%$ of gelatin), and (D) 0.20 solely gelatin $(20 \%$ gelatin $)$. 


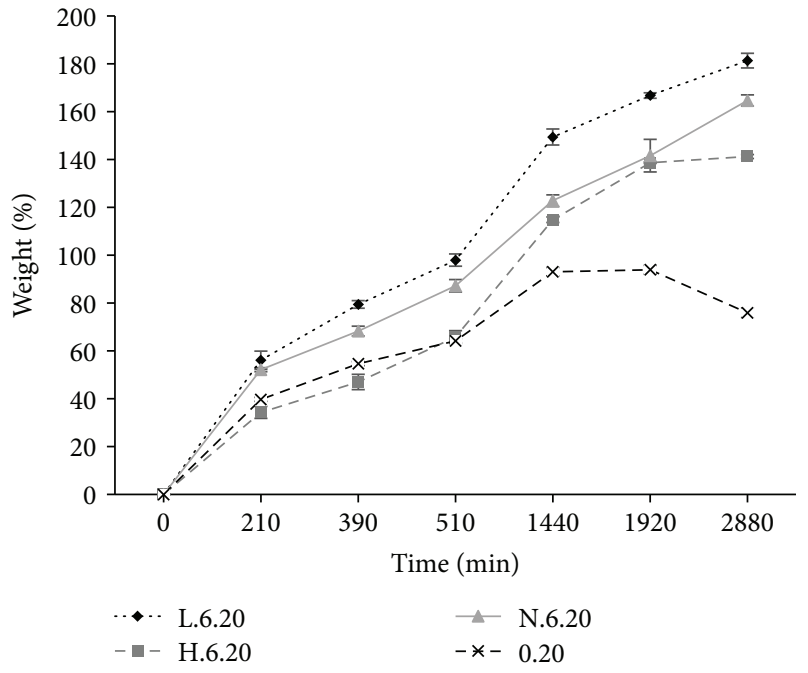

Figure 3: Swelling kinetics for four formulations of hydrogels incubated in PBS. The four samples were 0.20 solely gelatin $(20 \%$ gelatin), N.6.20 (6\% NOP and 20\% of gelatin), H.6.20 (6\% HOP and $20 \%$ of gelatin), and L.6.20 (6\% LOP and $20 \%$ gelatin).

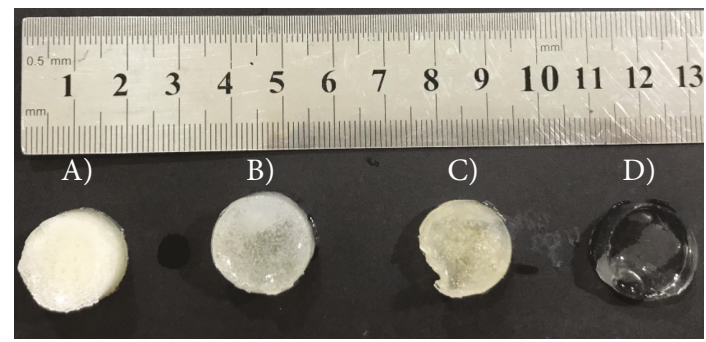

FIGURE 4: Hydrogels of gelatin and pectin, with different oxidation levels, after $72 \mathrm{~h}$ of swelling in a SBF at $32^{\circ} \mathrm{C}$. From left to right, the four samples are H.6.20 (6\% HOP and 20\% of gelatin), L.6.20 (6\% LOP and $20 \%$ of gelatin), N.6.20 (6\% NOP and $20 \%$ of gelatin), and 0.20 solely gelatin ( $20 \%$ gelatin).

changes the swelling properties of the hydrogel allowing higher swelling ratios, and equilibrium swelling is reached in longer periods of time.

The formulations that contain oxidized pectin exhibit higher swelling ratios compared to solely gelatin and native pectin hydrogels; previous works on pectin hydrogels propose that higher swelling in hydrogels is due to a high number of carboxyl groups present in pectin which makes it more hydrophilic [25]. It may also happen that the bonding of the carbonyl groups from the oxidized pectin with the gelatin's amine groups could bring more stability to the structure, via Schiff bases.

In Figure 4, the hydrogels after the swelling process are displayed. Sample 0.20 (control sample, only $20 \%$ gelatin) is the most deformed sample due to swelling; its cylindrical shape is changed to an amorphous one. The native pectin sample (N.6.20) is weakened during the swelling process, and it is very susceptible to ruptures upon handling. However, oxidized pectin hydrogels (H.6.20 and L.6.20) kept their cylindrical forms, but their radii increased due to swelling.

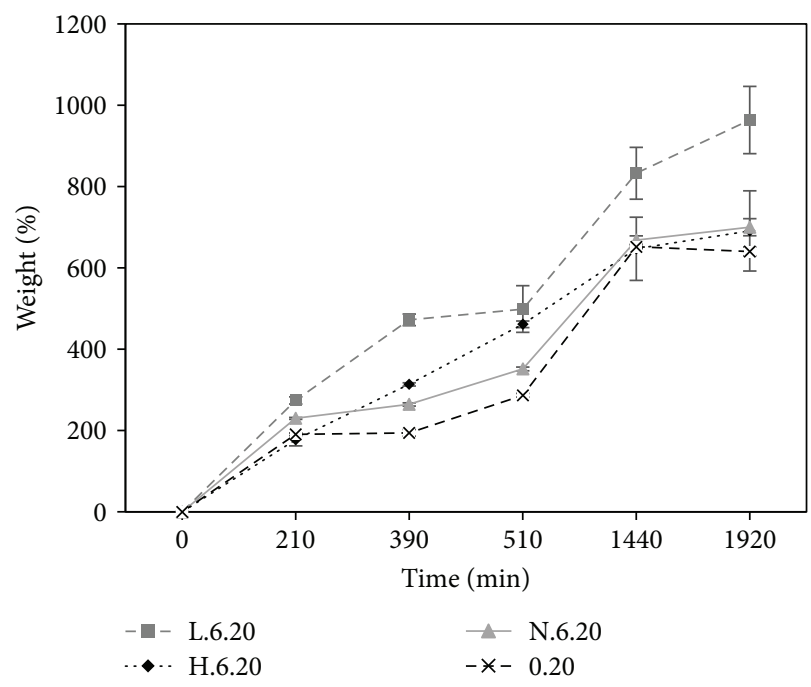

FIgURE 5: Swelling kinetics of aerogels (lyophilized hydrogels). The samples used for this study were H.6.20 (6\% HOP and $20 \%$ of gelatin), L.6.20 (6\% LOP and 20\% of gelatin), N.6.20 (6\% NOP and $20 \%$ of gelatin), and 0.20 solely gelatin ( $20 \%$ gelatin).

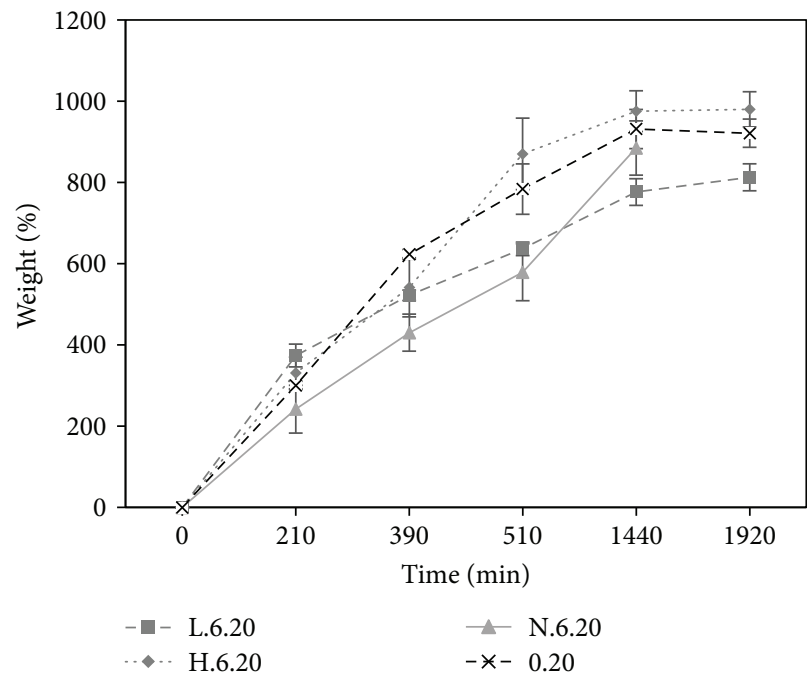

FIgURE 6: Swelling kinetics of xerogels. The samples used for this study were H.6.20 (6\% HOP and 20\% of gelatin), L.6.20 (6\% LOP and $20 \%$ of gelatin), N.6.20 (6\% NOP and $20 \%$ of gelatin), and 0.20 solely gelatin ( $20 \%$ gelatin). The weight of N.6.20 at $72 \mathrm{~h}$ was not obtained due to the fragmentation of the hydrogel.

Hydrogels that contain oxidized pectin are less deformed due to pectin properties that increase tensile force and work [26] as cross-linking with gelatin is more likely to take place as more functional groups are available.

3.2. Xerogel and Aerogel Swelling. The swelling of xerogels and aerogels is a test usually performed on the threedimensional structures obtained from the hydrogel to understand how the polymer chains interact with water; moreover, swelling of xero- and aerogels gives an idea of their morphology and pore distribution of the original hydrogel form [27]. 


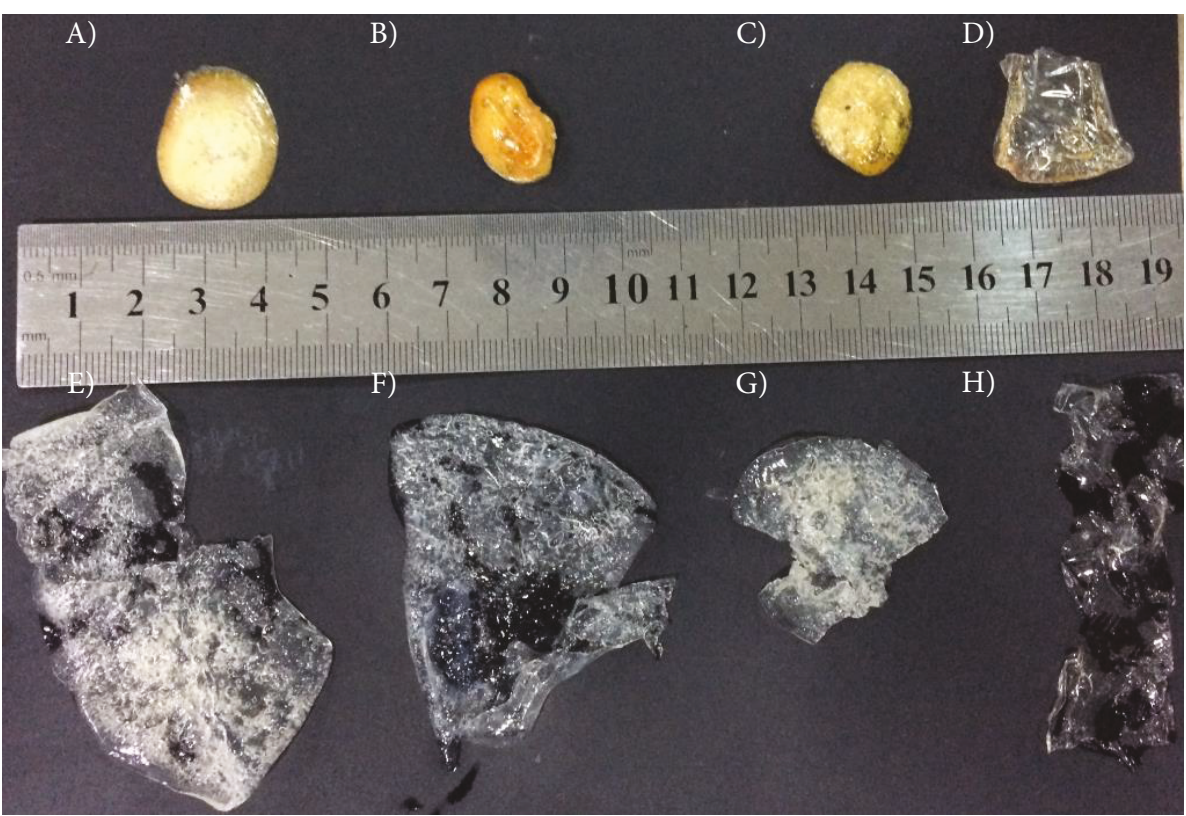

Figure 7: Swelling studies on xerogels. The samples in this figure are (A) dried H.6.20 (6\% HOP and 20\% of gelatin), (B) dried L.6.20 (6\% LOP and $20 \%$ of gelatin), (C) dried N.6.20 (6\% NOP and 20\% of gelatin), (D) dried 0.20 solely gelatin (20\% gelatin), (E) swelled H.6.20 (6\% HOP and $20 \%$ of gelatin), (F) swelled L.6.20 (6\% LOP and 20\% of gelatin), (G) swelled N.6.20 (6\% NOP and 20\% of gelatin), and (H) swelled 0.20 solely gelatin (20\% gelatin).

The following study was carried out in lyophilized and oven-dried hydrogels, in order to understand how temperature affects the hydrogel structure and swelling behavior.

The swelling process of the lyophilized hydrogels (aerogels) is presented in Figure 5. The aerogels that contain oxidized pectin, either high- or low-oxidation degree (H.6.20 and L.6.20), present a higher and faster swelling process, comparable to that of the hydrogels with native or no pectin. Native pectin aerogels and control samples (N.6.20 and 0.20, respectively) reach a maximum at 1440 minutes, shorter than the time for maximum swelling achieved by the oxidized pectin aerogels, which is after 1920 minutes.

Xerogels were obtained at $35^{\circ} \mathrm{C}$ until constant weight (approx. 10 hours) to avoid pectin damage due to temperature.

Figure 6 shows the swelling ratio over time of the oven-dried hydrogels, xerogels. It can be observed that HOP xerogels (H.6.20) can reach the highest swelling when dried by heating, compared to the highest swelling of aerogels. Moreover, sample L.6.20 achieved the lowest swelling degree when in xerogel form, a similar swelling behavior to its aerogel counterpart. This kinetics suggests that LOP hydrogels' structure may have not been greatly affected by temperature as the other samples, maintaining its swelling behavior.

Xerogels before and after the swelling process can be seen in Figure 7. The amorphous form may explain the considerable error bars shown in Figure 6. Xerogels reached higher swelling, when compared to the aerogel and hydrogel forms; this phenomenon might be attributed to the larger pores formed at higher temperatures during swelling. LOP and HOP have higher reswelling rates in both cases (xerogel and aerogel), perhaps due to the free carbonyl groups that are not part of the cross-linking that attract water molecules.

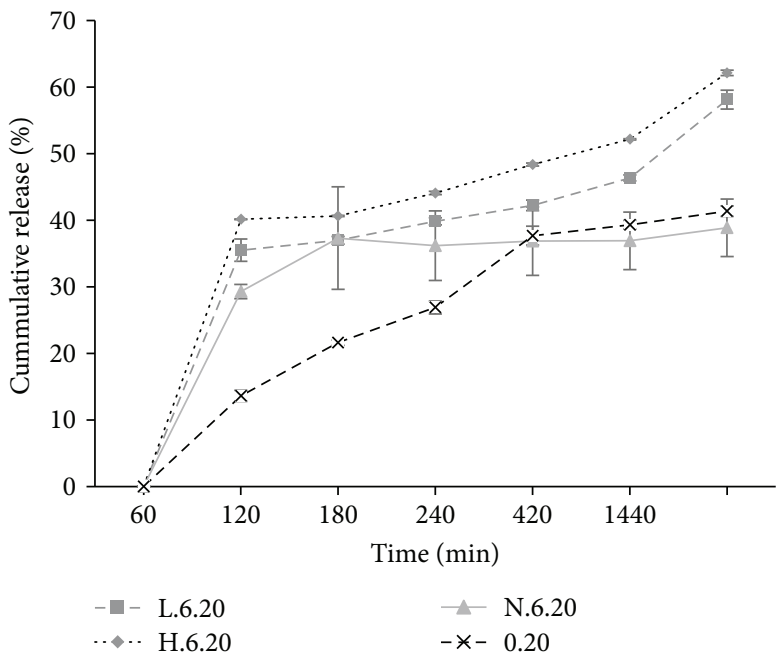

Figure 8: Drug release of acetaminophen from oxidized pectin-gelatin hydrogels. The cumulative amount of acetaminophen released is presented as function of time. The samples used for this analysis were H.6.20 (6\% HOP and 20\% of gelatin), L.6.20 (6\% LOP and $20 \%$ of gelatin), N.6.20 (6\% NOP and $20 \%$ of gelatin), and 0.20 solely gelatin ( $20 \%$ gelatin).

As it was reported previously by Chetouani et al., the gelatin pectin hydrogels have more thermal stability than the solely gelatin hydrogels [14]; native pectin hydrogels did not reach the final experiment time (1920 minutes) due to ruptures and fragmentation as is shown in Figure 7. Samples corresponding to low-oxidation-degree pectin, LOP (L.6.20), were the most resistant to temperature drying, to achieve the 
(a)

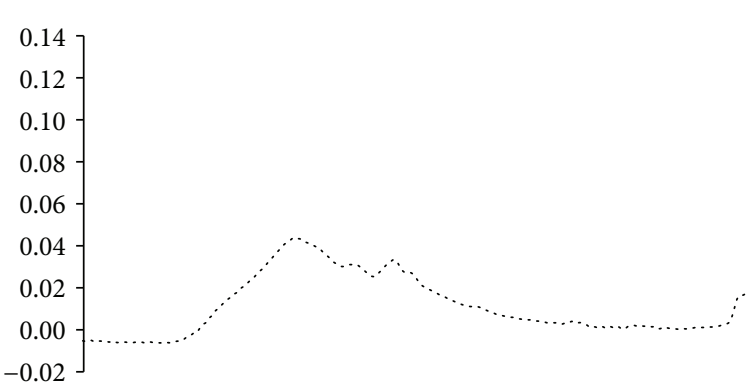

(b)

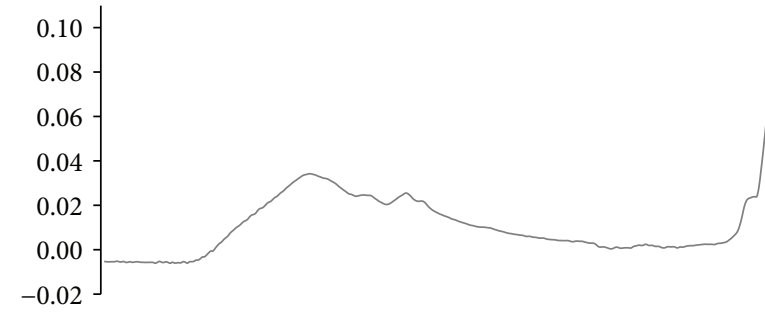

(c)

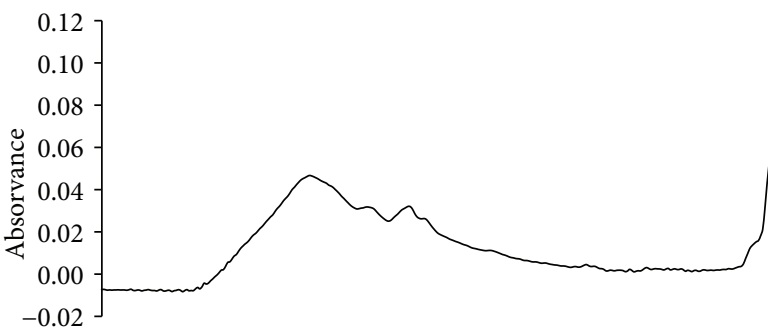

(d)

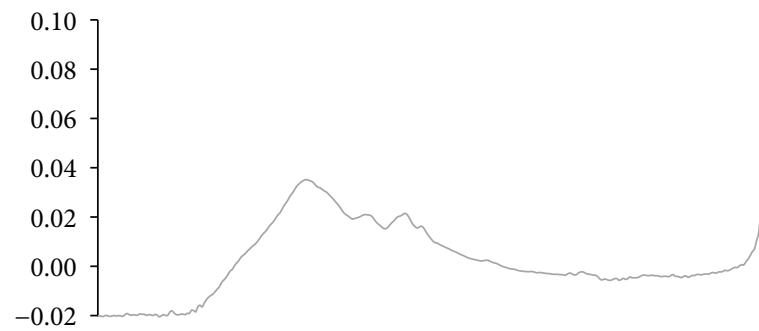

(e)

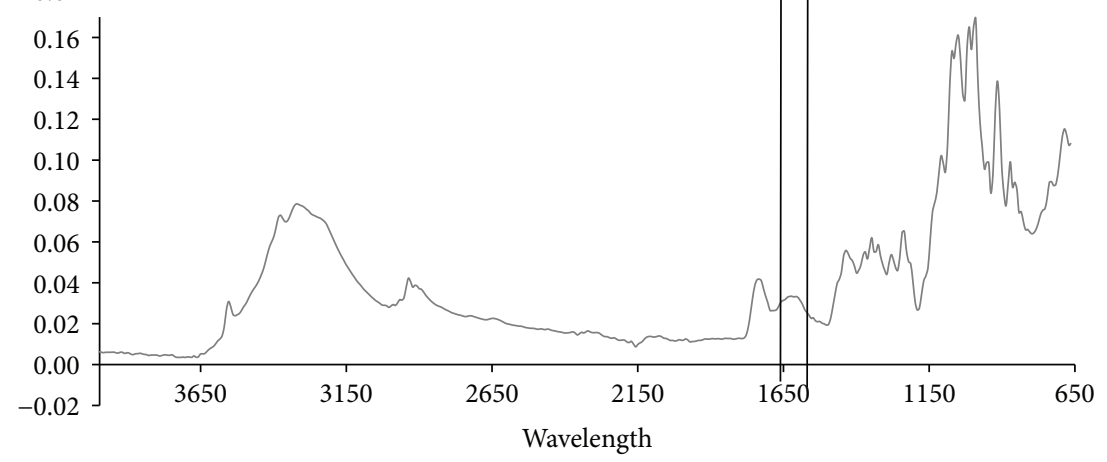

FIGURE 9: FTIR spectra of hydrogels. Absorbance is a wavelength function. The spectra shown in Figure 8 are (a) H.6.20 (6\% HOP and $20 \%$ of gelatin), (b) L.6.20 (6\% LOP and 20\% of gelatin), (c) N.6.20 (6\% NOP and 20\% of gelatin), (d) 0.20 solely gelatin (20\% gelatin), and (e) solely native pectin.

xerogel form, since their swelling behavior did not change significantly compared to other samples.

3.3. Drug Release. In this study, acetaminophen was used as a model molecule to understand the behavior of the hydrogel as a matrix for controlled drug delivery.
Figure 8 shows the release kinetics of acetaminophen from the hydrogels for up to 32 hours. Oxidized pectin hydrogels (H.6.20 and L.6.20) displayed higher release rates; the highest amount released was $62.13 \pm 0.40 \%$ for the HOP hydrogel (H.6.20). The release rates from NOP and sole gelatin hydrogels were similar but lower than those achieved by the systems based on oxidized pectin. 


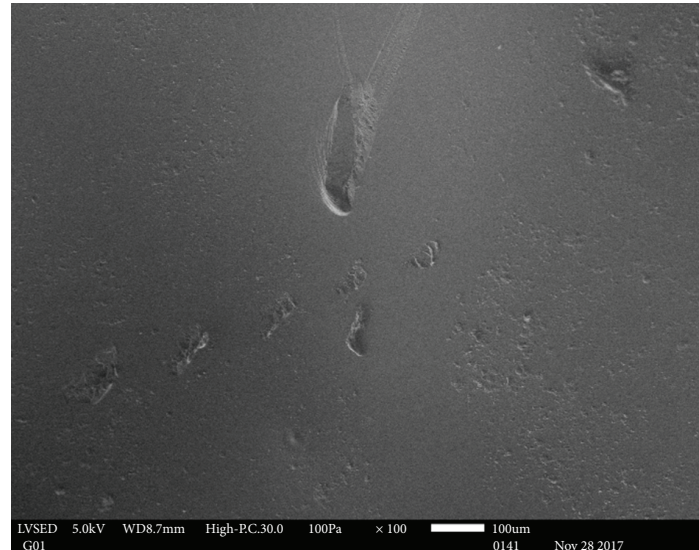

(a)

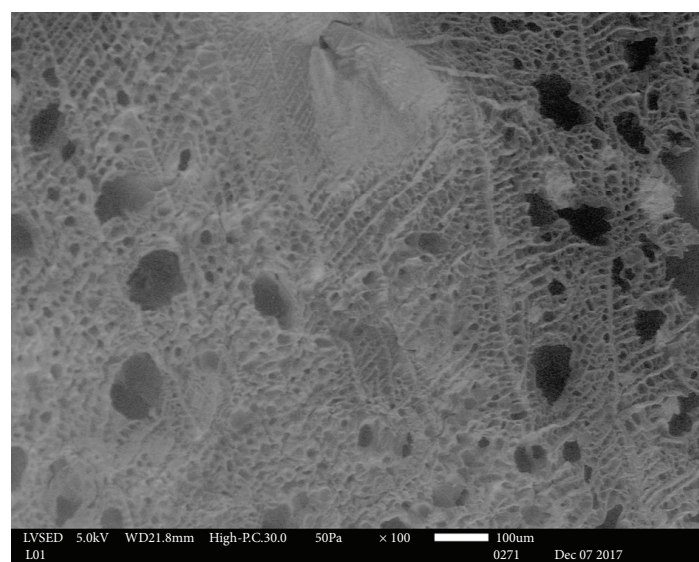

(c)

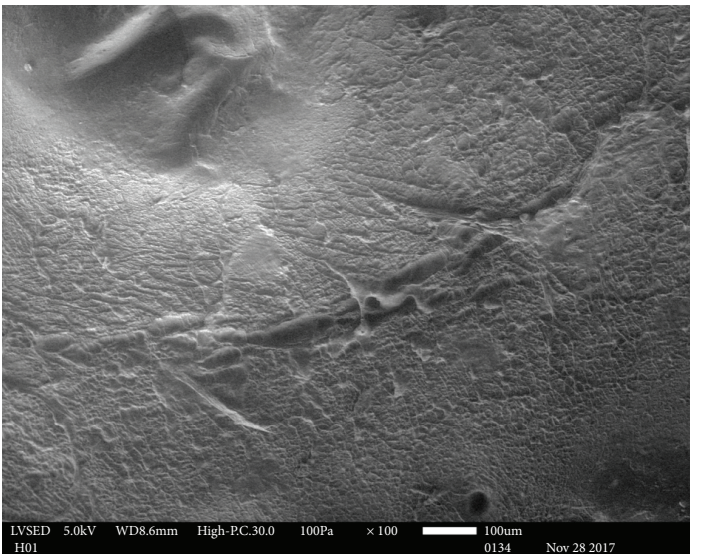

(b)

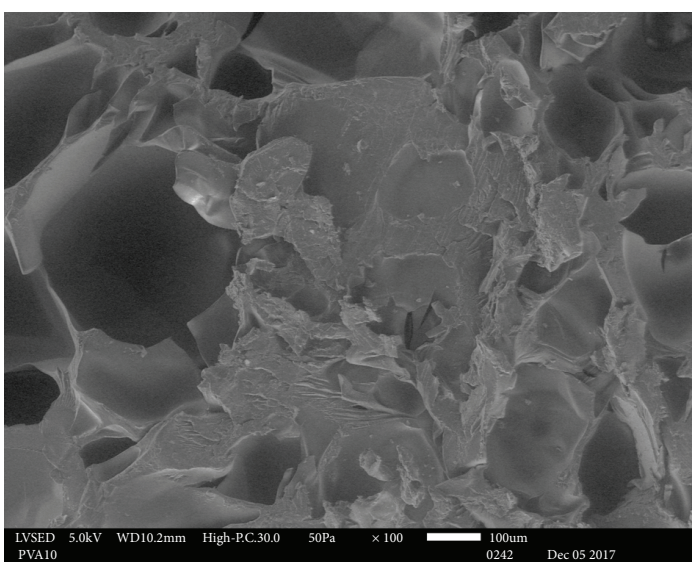

(d)

FIGURE 10: Scanning electron micrographs of hydrogels at $100 \mathrm{x}$ resolution. The samples used for this analysis were (a) 0.20 solely gelatin (20\% gelatin), (b) H.6.20 (6\% HOP and 20\% of gelatin), (c) L.6.20 (6\% LOP and 20\% of gelatin), and (d) N.6.20 (6\% NOP and $20 \%$ of gelatin).

The higher release rates of oxidized pectin hydrogels could be caused by the homogeneous distribution of the drug in the hydrogel, when compared to solely gelatin hydrogels that promote the creation of acetaminophen crystals and heterogeneous distribution of the drug. Furthermore, the swelling tests previously performed show that the solvent diffusion among the oxidized pectin hydrogels is high, as these samples have the highest swelling, which in turn translate into higher drug release percentages.

Even though there is no previous research on drug release kinetics using oxidized pectin-gelatin hydrogels, there are some studies that may allow comparison of these results. Previous studies on modified pectin as a drug carrier of water-insoluble drugs show low release or noncontrolled release at pH 5.0 [28]; however, hydrogels shown in the present work display a controlled delivery, as the maximum release is reached at 72 hours. Moreover, studies on gelatin capsules report material disintegration within 10 minutes, which is classified as fast release [29]. This suggests that there may be important material-drug interactions happening in the OP-gelatin hydrogels which may result in more sustained release.
3.4. FTIR Spectra. Spectra of the four samples previously mentioned (H.6.20, L.6.20, N.6.20, and 0.20 and native pectin) are shown in Figure 9.

The FTIR of the hydrogels (N.6.20, L.6.20, and H.6.20) and gelatin are very similar, as the percentage of gelatin is higher than that of pectin. However, there is a displacement of some peaks at the N.6.20 sample, probably due to the interactions between native pectin and gelatin; for example, pectin's peak at $1727 \mathrm{~cm}^{-1}$ which corresponds to free carbonyl groups [30] and can be seen in Figure 9(e) is not visible in other FTIR spectra due to the possible interactions these groups may have. Samples of oxidized pectin (L.6.20 and H.6.20) present a more complex matrix, as the oxidation procedure alters the structure of pectin creating diverse residues.

The interactions in H.6.20 and L.6.20 can occur probably due to a Schiff base formation as a thin band around $1630 \mathrm{~cm}^{-1}$ [31], suggesting that there are some interactions between the $\mathrm{C}=\mathrm{N}$ groups. A band around $1734 \mathrm{~cm}^{-1}$ [32] was expected, as a result of the oxidation process; however, the functionalized groups are the ones responsible for the gelatin cross-linking. 


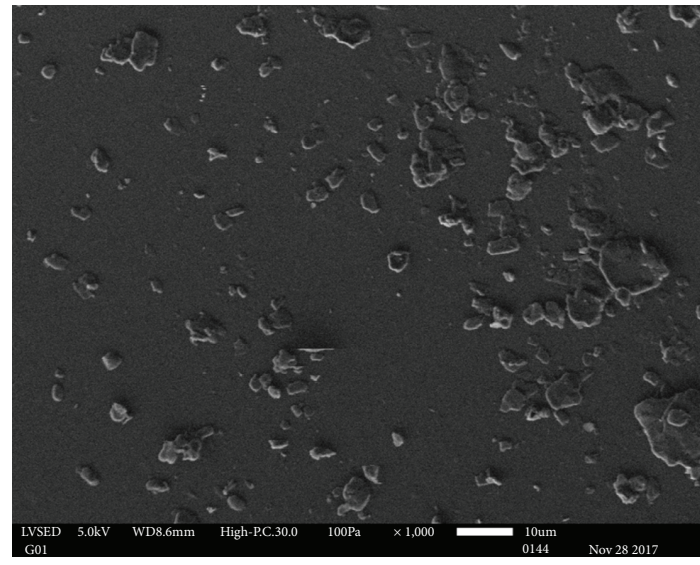

(a)

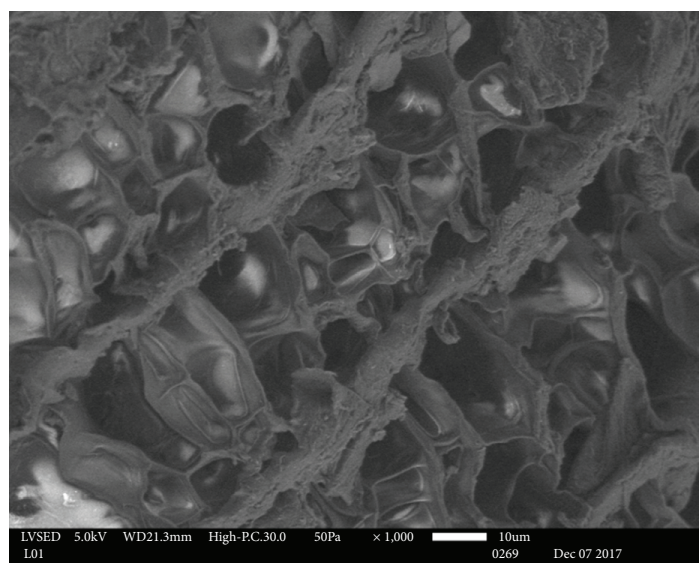

(c)

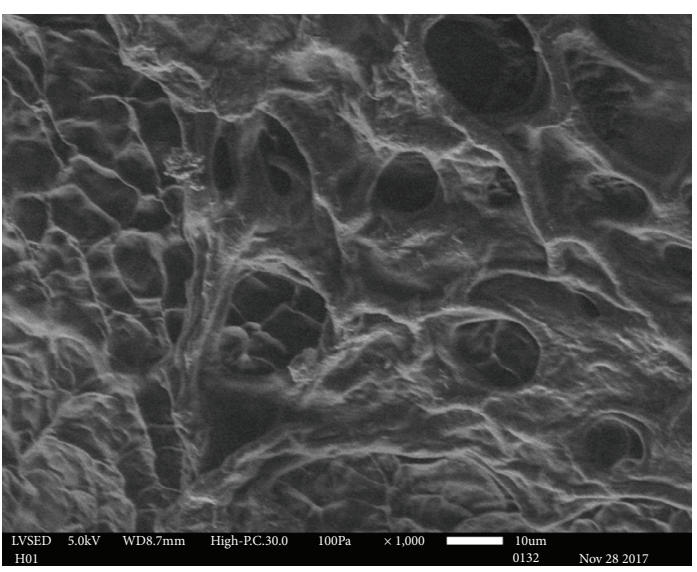

(b)

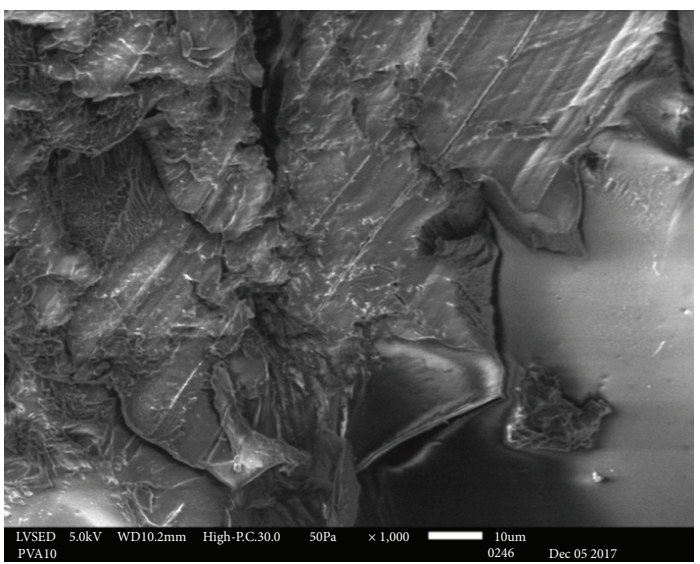

(d)

FIGURE 11: Scanning electron micrographs of hydrogels at $1 \mathrm{~K}$ resolution. The samples used for this analysis were (a) 0.20 solely gelatin (20\% gelatin), (b) H.6.20 (6\% HOP and 20\% of gelatin), (c) L.6.20 (6\% LOP and 20\% of gelatin), and (d) N.6.20 (6\% NOP and $20 \%$ of gelatin).

3.5. SEM. Figures 10 and 11 present SEM images for different hydrogel formulations, in which the pore size and organization differ from one another. The sole gelatin sample, shown in Figures 10(a) and 11(a), presented a mainly flat surface, with some lumps appreciable; nonetheless, no pores are identified.

Pectin gelatin materials have different pore structures, as well. Native pectin-gelatin hydrogels present an irregular structure, which can be seen in the SEM in Figure 11 at $1 \mathrm{~K}$ resolution which does not allow the pores to be seen as they are bigger than $100 \mu \mathrm{m}$. Low-oxidation pectin-gelatin hydrogels have well-organized pores, mostly smaller than $10 \mu \mathrm{m}$, and high-oxidation pectin-gelatin hydrogels present nonstructured pores, which have several sizes that range from $2 \mu \mathrm{m}$ to $15 \mu \mathrm{m}$.

Figure 11 shows the SEM analysis at 100x resolution. The solely gelatin sample presents a smooth surface compared to the other hydrogels. Native pectin (Figure 11(b)) presents a rough surface; however, no pores are shown. In contrast, oxidized pectin pictures present numerous pores in their structure; low-oxidation pectin hydrogel (Figure 11(c)) has numerous pores in an organized structure, and high-oxidation pectin (Figure 11(d)) presents disorganized, different-sized pores, with diameters ranging from $150 \mu \mathrm{m}$ to $10 \mu \mathrm{m}$.

The HOP and LOP hydrogels are the most porous structures, and this may be the explanation of their characteristics. High swelling and sustained drug release are also a result of their uniform pore distribution, as the storage of the drug can occur inside the pores and also allows solvent diffusion [33].

\section{Conclusions}

The oxidized pectin-gelatin hydrogels present numerous characteristics that make them an alternative for wound dressings and controlled drug delivery matrices. Swelling and reswelling (from xerogel and aerogel forms) showed that OP-gelatin hydrogels have improved swelling ratios and stability compared to NOP and solely gelatin; this may be due to their porous structures as SEM studies showed. The porosity of the OP hydrogels allowed having sustained and high release rates, which make them an option for wound dressing. In order to confirm the application of OP-gelatin hydrogels as wound dressings, mechanical characterization and biocompatibility tests should be performed. 


\section{Data Availability}

Data from the present study is available upon request to the corresponding author.

\section{Conflicts of Interest}

The authors declare that there is no conflict of interest regarding the publication of this paper.

\section{Acknowledgments}

The present study was funded by the PoliGrant program (10133) and Collaboration Grant program (11163) at the Universidad San Francisco de Quito. The authors would like to acknowledge the Laboratory of Environmental Engineering-USFQ for allowing part of the experiments to be conducted in their facilities.

\section{References}

[1] A. Sionkowska, "Current research on the blends of natural and synthetic polymers as new biomaterials: review," Progress in Polymer Science, vol. 36, no. 9, pp. 1254-1276, 2011.

[2] G. F. Paciotti, L. Myer, D. Weinreich et al., "Colloidal gold: a novel nanoparticle vector for tumor directed drug delivery," Drug Delivery, vol. 11, no. 3, pp. 169-183, 2008.

[3] M. Prabaharan, "Review paper: chitosan derivatives as promising materials for controlled drug delivery," Journal of Biomaterials Applications, vol. 23, no. 1, pp. 5-36, 2008.

[4] F. L. Seixas, D. L. Fukuda, F. R. B. Turbiani et al., "Extraction of pectin from passion fruit peel (Passiflora edulis f. flavicarpa) by microwave-induced heating," Food Hydrocolloids, vol. 38, pp. 186-192, 2014.

[5] B. R. Thakur, R. K. Singh, A. K. Handa, and M. A. Rao, "Chemistry and uses of pectin - a review," Critical Reviews in Food Science and Nutrition, vol. 37, no. 1, pp. 47-73, 1997.

[6] J. Rose, S. Pacelli, A. Haj et al., "Gelatin-based materials in ocular tissue engineering," Materials, vol. 7, no. 4, pp. 3106-3135, 2014.

[7] B. Gupta, M. Tummalapalli, B. L. Deopura, and M. S. Alam, "Preparation and characterization of in-situ crosslinked pectin-gelatin hydrogels," Carbohydrate Polymers, vol. 106, pp. 312-318, 2014.

[8] Y. Tabata and Y. Ikada, "Protein release from gelatin matrices," Advanced Drug Delivery Reviews, vol. 31, no. 3, pp. 287-301, 1998.

[9] T. W. Wong, G. Colombo, and F. Sonvico, "Pectin matrix as oral drug delivery vehicle for colon cancer treatment," AAPS PharmSciTech, vol. 12, no. 1, pp. 201-214, 2011.

[10] L. Liu, M. L. Fishman, J. Kost, and K. B. Hicks, "Pectin-based systems for colon-specific drug delivery via oral route," Biomaterials, vol. 24, no. 19, pp. 3333-3343, 2003.

[11] S. Van Vlierberghe, E. Vanderleyden, V. Boterberg, and P. Dubruel, "Gelatin functionalization of biomaterial surfaces: strategies for immobilization and visualization," Polymers, vol. 3, no. 1, pp. 114-130, 2011.

[12] A. Bigi, E. Boanini, S. Panzavolta, and N. Roveri, "Biomimetic growth of hydroxyapatite on gelatin films doped with sodium polyacrylate," Biomacromolecules, vol. 1, no. 4, pp. 752-756, 2000.
[13] T. Yoshimura, K. Sengoku, and R. Fujioka, "Pectin-based surperabsorbent hydrogels crosslinked by some chemicals: synthesis and characterization," Polymer Bulletin, vol. 55, no. 1-2, pp. 123-129, 2005.

[14] A. Chetouani, M. Elkolli, M. Bounekhel, and D. Benachour, "Synthesis and properties of novel hydrogels from oxidized pectin crosslinked gelatin for biomedical applications," Polymer Bulletin, vol. 71, no. 9, pp. 2303-2316, 2014.

[15] W. J. Rogers, "Sterilisation techniques for polymers," Sterilisation of Biomaterials and Medical Devices, pp. 151211, 2012, https://www.sciencedirect.com/science/article/pii/ B9781845699321500076.

[16] H. Zhao and N. D. Heindel, "Determination of degree of substitution of formyl groups in hydroxylamine hydrochloride method," Pharmaceutical Research, vol. 08, no. 3, pp. 400402, 1991.

[17] R. K. Mishra, A. B. A. Majeed, and A. K. Banthia, "Development and characterization of pectin/gelatin hydrogel membranes for wound dressing," International Journal of Plastics Technology, vol. 15, no. 1, pp. 82-95, 2011.

[18] T. Begam, A. K. Nagpal, and R. Singhal, "A comparative study of swelling properties of hydrogels based on poly(acrylamide-co-methyl methacrylate) containing physical and chemical crosslinks," Journal of Applied Polymer Science, vol. 89, no. 3, pp. 779-786, 2003.

[19] V. R. Patel and M. M. Amiji, "Preparation and characterization of freeze-dried chitosan-poly (ethylene oxide) hydrogels for site-specific antibiotic delivery in the stomach," Pharmaceutical Research, vol. 13, no. 4, pp. 588-593, 1996.

[20] B. Gaihre, M. Khil, D. Lee, and H. Kim, "Gelatin-coated magnetic iron oxide nanoparticles as carrier system: drug loading and in vitro drug release study," International Journal of Pharmaceutics, vol. 365, no. 1-2, pp. 180-189, 2009.

[21] M. C. Meyer, A. B. Straughn, R. M. Mhatre et al., "The effect of gelatin cross-linking on the bioequivalence of hard and soft gelatin acetaminophen capsules," Pharmaceutical Research, vol. 17, no. 8, pp. 962-966, 2000.

[22] P. T. S. Kumar, C. Ramya, R. Jayakumar, S. K. V. Nair, and V. K. Lakshmanan, "Drug delivery and tissue engineering applications of biocompatible pectin-chitin/nano $\mathrm{CaCO}_{3}$ composite scaffolds," Colloids and Surfaces B: Biointerfaces, vol. 106, pp. 109-116, 2013.

[23] B. Balakrishnan, A. Jayakrishnan, S. S. P. Kumar, and A. M. Nandkumar, "Anti-bacterial properties of an in situ forming hydrogel based on oxidized alginate and gelatin loaded with gentamycin," Biomaterials and Artificial Organs, vol. 26, no. 3, pp. 139-145, 2012.

[24] M. Pinelo, B. Zeuner, and A. S. Meyer, "Juice clarification by protease and pectinase treatments indicates new roles of pectin and protein in cherry juice turbidity," Food and Bioproducts Processing, vol. 88, no. 2-3, pp. 259-265, 2010.

[25] A. Akhgari, F. Farahmand, H. A. Garekani, F. A. Sadeghi, and T. Vandamme, "The effect of pectin on swelling and permeability characteristics of free films containing Eudragit RL and / or RS as a coating formulation aimed for colonic drug delivery," Daru: Journal of Faculty of Pharmacy, Tehran University of Medical Sciences, vol. 18, no. 2, pp. 91-96, 2010.

[26] C. Phimpharian, A. Jangchud, K. Jangchud, N. Therdthai, W. Prinyawiwatkul, and H. K. No, "Physicochemical characteristics and sensory optimisation of pineapple leather snack as affected by glucose syrup and pectin concentrations," 
International Journal of Food Science and Technology, vol. 46, no. 5, pp. 972-981, 2011.

[27] R. Gemeinhart and C. Guo, "Fast swelling hydrogel systems," in Reflexive Polymers and Hydrogels: Understanding and Designing Fast Responsive Polymeric Systems, pp. 245-257, CRC Press, 2004.

[28] O. Munjeri, J. H. Collett, and J. T. Fell, "Hydrogel beads based on amidated pectins for colon-specific drug delivery: the role of chitosan in modifying drug release," Journal of Controlled Release, vol. 46, no. 3, pp. 273-278, 1997.

[29] J. Brown, N. Madit, E. T. Cole, I. R. Wilding, and D. Cadé, "The effect of cross-linking on the in vivo disintegration of hard gelatin capsules," Pharmaceutical Research, vol. 15, no. 7, pp. 1026-1030, 1998.

[30] M. d. F. Sato, D. C. Rigoni, M. H. G. Canteri, C. L. d. O. Petkowicz, A. Nogueira, and G. Wosiacki, "Chemical and instrumental characterization of pectin from dried pomace of eleven apple cultivars," Acta Scientiarum Agronomy, vol. 33, no. 3, 2011.

[31] Jenisha, S. Theodore David, and J. P. Priyadharsini, "Schiff base ligand its complexes and their FT-IR spectroscopy studies," International Journal on Applied Bio-Engineering, vol. 9, no. 1, pp. 1-4, 2015.

[32] B. Gupta, M. Tummalapalli, B. L. Deopura, and M. S. Alam, "Functionalization of pectin by periodate oxidation," Carbohydrate Polymers, vol. 98, no. 1, pp. 1160-1165, 2013.

[33] M. E. Davis, "Ordered porous materials for emerging applications," Nature, vol. 417, no. 6891, pp. 813-821, 2002. 


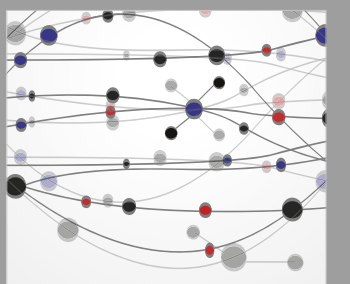

The Scientific World Journal
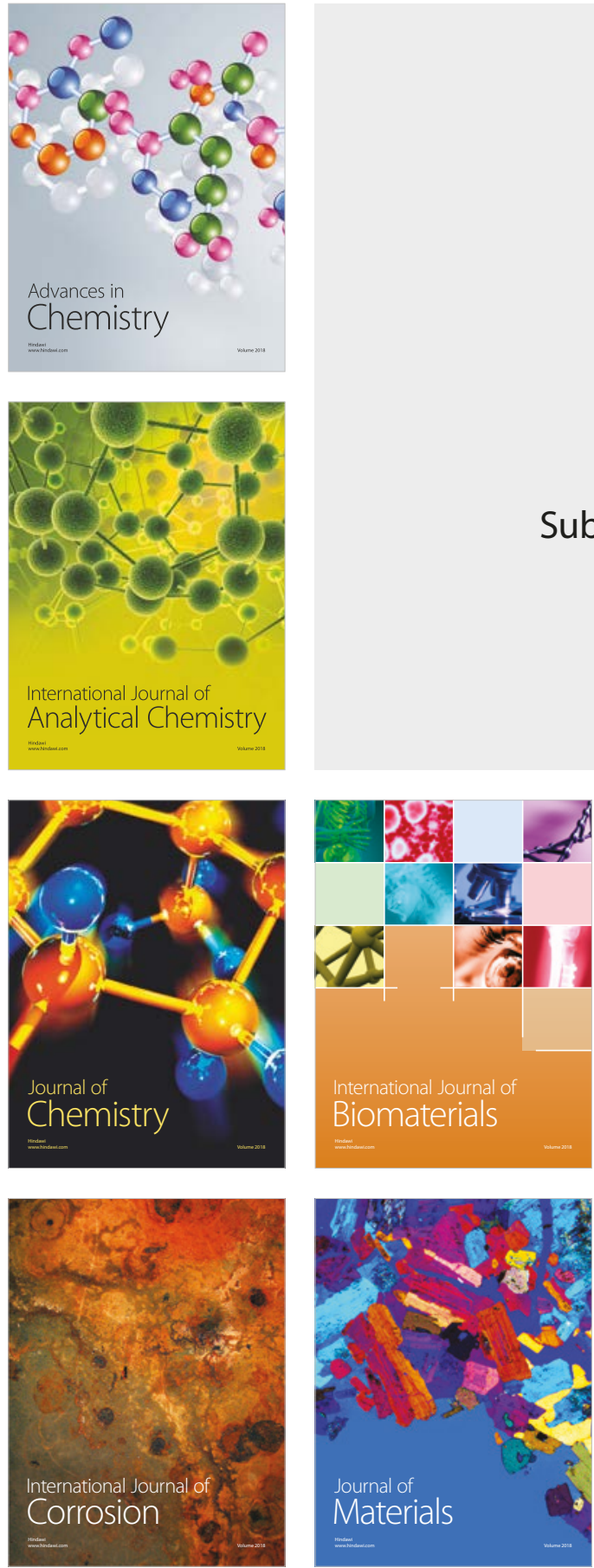

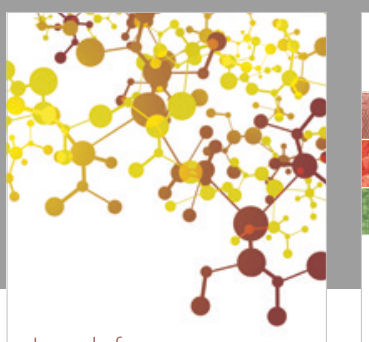

Journal of

Applied Chemistry
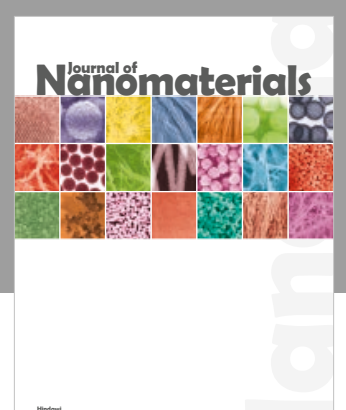

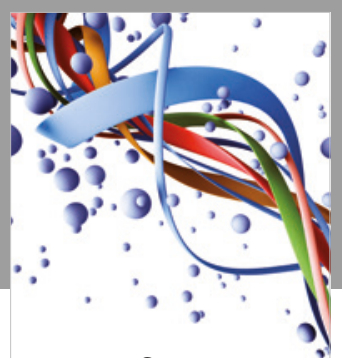

Scientifica

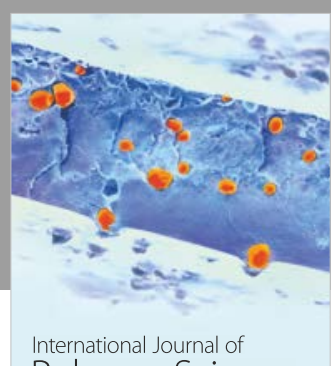

Polymer Science

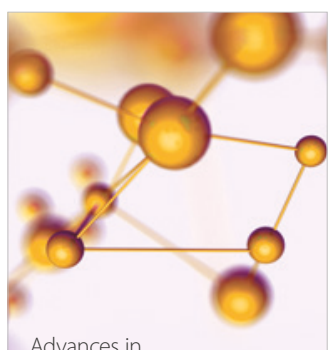

Physical Chemistry
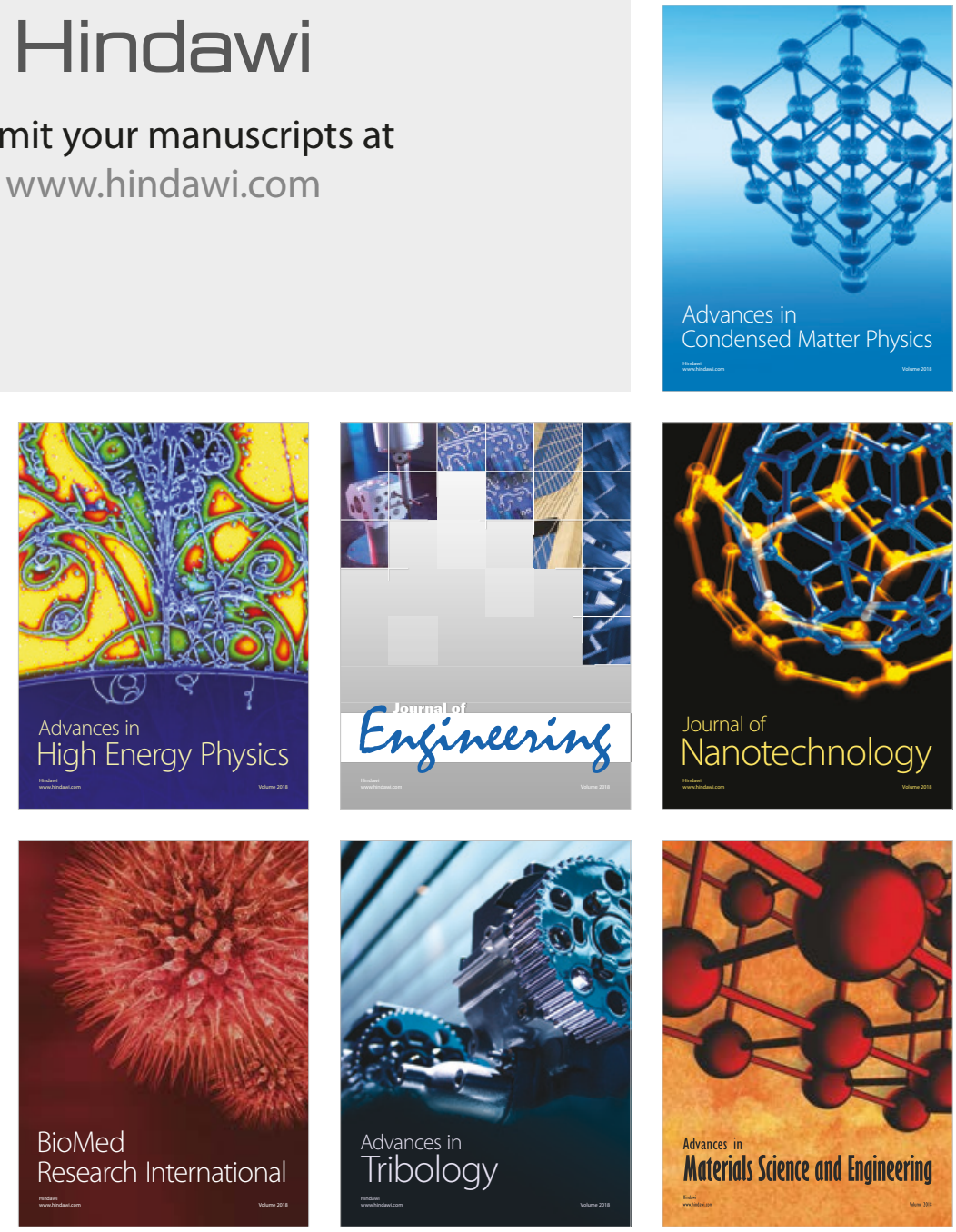